\section{Condições de saúde bucal de crianças na faixa etária pré-escolar, residentes em áreas de abrangência do Programa Saúde da Família em Salvador, Bahia, Brasil}

\author{
The dental health of preschool-aged \\ children resident in areas covered by the \\ Family Health Program, in the city of \\ Salvador, in the State of Bahia, Brazil
}

Abstract

Objectives: to determine the prevalence and severity of dental caries, malocclusions and gum damage in five-year-old preschool children resident in areas covered by the Family Health Program (PSF).

Methods: a cross-cutting study was carried out with five-year-old children examined by PSF dental health teams and as part of the Collective Procedures Program of the municipal health service of the city of Salvador, in the State of Bahia, Brazil, in 2005. The diagnostic criteria established by the World Health Organization and the Dental Health Project were used and the examinations were carried out in schools, crèches or the children's homes.

Results: the investigation involved 1,374 children. The prevalence of caries was $49.6 \%$ and the def-d (average number of decayed, extracted or filled teeth) 1.97, most of these being cases of caries (85.78\%). The prevalence of gum damage was $7.40 \%$ and for slight and moderate/severe malocclusion, $24.90 \%$ and $12.70 \%$, respectively.

Conclusions: there was a high prevalence of severe dental problems that are easy to prevent and control by way of more effective educational, preventive and curative action during primary care for preschool-aged children in the municipality. The service's epidemiology department could help to plan and oversee such action.

Key words Dental caries, Gingival diseases, Malocclusion, Oral health, Child, preschool
Tatiana Frederico de Almeida 1

Maria Cristina Teixeira Cangussu 2

Sônia Cristina Lima Chaves 3

David Inácio de Castro e Silva 4

Sóstenes Conceição dos Santos 5

1-3 Faculdade de Odontologia. Universidade Federal da Bahia Avenida Araújo Pinho, n. 62. Salvador, BA, Brasil. CEP: 40.100000. E-mail: tatifrederico@hotmail.com

4,5 Instituto de Saúde Coletiva. Universidade Federal da Bahia. Salvador, Bahia, Brasil

\section{Resumo}

Objetivos: determinar a prevalência e gravidade da cárie dentária, maloclusões, e alteração gengival em pré-escolares de cinco anos de idade residentes em áreas do Programa Saúde da Família (PSF).

Métodos: estudo de corte transversal, conduzido com crianças de cinco anos examinadas por equipes de saúde bucal do PSF e do programa de Procedimentos Coletivos do serviço municipal de saúde de Salvador, Bahia, Brasil, em 2005. Os critérios de diagnóstico da Organização Mundial da Saúde e do Projeto Saúde Bucal foram utilizados e os exames foram realizados em escolas, creches ou em domicílios.

Resultados: participaram deste inquérito 1374 crianças. A prevalência da cárie foi de 49,6\% e o ceo$d$ (média de dentes cariados, extraidos e obturados) igual a 1,97, sendo que a maioria dos dentes encontrava-se cariados $(85,78 \%)$. A prevalência de alteração gengival foi de 7,40\% e maloclusões leve e moderada/severa de $24,90 \%$ e $12,70 \%$, respectivamente.

Conclusões: foram constatadas elevada prevalencia e gravidade de problemas bucais passiveis de prevenção e controle mediante ações educativas, preventivas e curativas mais efetivas da atenção primária, em pré-escolares no município. A epidemiologia no serviço pode colaborar para o planejamento e acompanhamento de tais ações Palavras-chave Cárie dentária, Doenças da gengiva, Maloclusão, Saúde bucal, Pré-escolares 


\section{Introdução}

No Brasil foram realizados três inquéritos nacionais de saúde bucal abrangendo crianças na faixa etária pré-escolar. 1-3 O último deles, finalizado em 2004 e denominado Projeto Saúde Bucal Brasil (SB Brasil), identificou um valor médio de ceo-d (média de dentes cariados, extraídos e obturados por cárie na dentição decídua) de 2,80 nesta população, porém com acentuadas diferenças entre regiões. ${ }^{3}$

Sabe-se que o comportamento da cárie na população pré-escolar varia de acordo com fatores relacionados ao estilo de vida, realidade socioeconômica e demográfica e acesso aos serviços de saúde.4-11 Sendo assim, algumas iniciativas vem sendo desenvolvidas nos serviços de atenção básica à saúde, visando identificar e assistir grupos populacionais ou famílias com situações especiais de vulnerabilidade para o desenvolvimento de problemas de saúde bucal.4,5,12,13

Na cidade de Salvador, poucos são os levantamentos de saúde bucal realizados. Barros et al. 14 investigando a ocorrência de cárie em crianças de 0 a 30 meses residentes no município, obteve uma prevalência de $55,3 \%$, que variou de $25 \%$, nas crianças com até um ano de idade, a $71 \%$, naquelas entre 25 e 30 meses de idade. Nesse levantamento, destacou-se a alta prevalência de cárie nessa população, que atingiu nível distante da meta de 50\% crianças de cinco a seis anos livres de cárie para o ano 2000, estabelecida pela Organização Mundial da Saúde (OMS). ${ }^{15}$ Quanto às alterações gengivais, cerca de $6,0 \%$ de crianças avaliadas no último levantamento nacional apresentaram este agravo bucal e cerca de $37 \%$ possuía algum tipo de maloclusão (leve ou moderada/severa). ${ }^{3}$

Neste sentido, o objetivo deste trabalho foi estimar a prevalência e gravidade da cárie dentária, alteração gengival e maloclusão na idade de cinco anos, em áreas cobertas pelas Equipes de Saúde Bucal (ESB) do Programa Saúde da Família (PSF) e áreas com atividades de procedimentos coletivos na atenção básica tradicional nos distritos sanitários onde ainda não existia implantação do PSF no município de Salvador.

\section{Métodos}

A população de estudo foi composta por crianças com cinco anos de idade, selecionadas de préescolas e domicílios localizados nas áreas de abrangência das equipes de saúde de bucal (ESB) do Programa de Saúde da Família (PSF), além de crianças assistidas pelas atividades de procedimentos coletivos nos distritos sanitários (DS) sem cobertura das equipes de saúde bucal (Centro Histórico e DS Boca do Rio).

O município de Salvador está dividido em doze distritos sanitários e possuía, em 2005, uma população estimada de 211.141 crianças até cinco anos de idade. $16 \mathrm{O}$ processo de implantação do PSF no município teve seu início no final da década de noventa no DS Subúrbio Ferroviário, que apresentava as piores condições sanitárias e socioeconômicas. Em 2005, o PSF não só assistia a maior parte da população desse distrito como havia se expandido para outras regiões da cidade, embora a cobertura do Programa de Saúde Bucal fosse ainda baixa em relação à população total do município. No período havia $35 \mathrm{USF}$, nas quais atuavam 79 equipes de profissionais de saúde bucal, representando uma cobertura de cerca de $30 \%$ desses serviços. Além das ESB, o município dispunha igualmente de um programa de Procedimentos Coletivos de Saúde Bucal que realizava semanalmente ações preventivas e educativas em escolas públicas de alguns distritos. Nos DS do Centro Histórico e Boca do Rio, os quais não constituíam áreas de cobertura das ESB, a seleção e o exame das crianças para o estudo foram realizados em três escolas e/ou creches assistidas pelo Programa de Procedimentos Coletivos de Saúde Bucal.

O cálculo da amostra e a seleção dos participantes foram efetuados com base na estratégia proposta pela OMS17 para levantamentos de saúde bucal em áreas com níveis de prevalência de cárie e doença periodontal baixo a moderado, uma vez que levantamentos anteriores de cárie dentária apontavam tais níveis de prevalência na população escolar em Salvador.3,14 Dessa forma, o tamanho da amostra foi calculado em 40 indivíduos por área de abrangência de cada ESB, os quais foram selecionados por meio de sorteio aleatório simples utilizando a listagem prévia de todas as crianças com cinco anos completos cadastradas nos programas.

Os pais ou responsáveis pelos participantes foram informados sobre os objetivos do estudo e as técnicas a serem realizadas durante o exame bucal e solicitada a autorização por escrito. Quando necessário, as crianças eram encaminhadas para atendimento odontológico na USF ou unidades básicas de saúde (UBS). O projeto foi submetido e aprovado pelo Comitê de Ética em Pesquisa do Hospital Universitário Professor Edgard Santos da Universidade Federal da Bahia (CEP 056-06/CEPHUPES).

A coleta dos dados foi realizada pelas $35 \mathrm{ESB}$ existentes no município, que são compostas por um 
cirurgião-dentista, um auxiliar de consultório dentário (ACD) e seis agentes comunitários de saúde (ACS), além de três cirurgiões-dentistas e três ACD's que atuavam no programa de Procedimentos Coletivos. O cirurgião-dentista, além de efetuar o exame clínico, realizou a implementação e supervisão das atividades de coleta das informações. Um dos ACD ou um ACS de cada ESB previamente treinado realizou o registro dos dados.

Visando a padronização dos procedimentos clínicos e dos critérios diagnósticos foi realizado o treinamento teórico dos cirurgiões-dentistas utilizando textos e fotos como recursos. Em seguida, a calibração do exame antes do início do inquérito foi efetuada, por meio do exame de 100 alunos com idade entre cinco a seis anos. Nessa etapa do estudo, os examinadores trabalharam em duplas ou trios. Cada um dos profissionais realizou em torno de 15 exames bucais, os quais incluíram a avaliação das afecções bucais de interesse para o estudo, tendo-se obtido uma concordância geral inter-examinador de 95\% para o diagnóstico da cárie dentária e cerca de $80 \%$ para as outras condições. Esses níveis de concordância são considerados satisfatórios para levantamentos epidemiológicos. A concordância intra-examinador não foi avaliada neste estudo.

$\mathrm{O}$ exame clínico visual foi realizado à luz natural, com uso de espátulas de madeira descartáveis e gaze para secar as unidades dentárias. Dados sobre o local da coleta dos dados (domicílio ou creche), o ceo-d, total de dentes decíduos examinados foram obtidos no momento do exame, de acordo com a metodologia da OMS.17 Avaliou-se igualmente a presença de alteração gengival e maloclusão, de acordo com os critérios dos índices adotados no SB Brasil, 3 índice de Alteração Gengival, que avalia presença de sangramento gengival espontâneo em pelo menos três unidades após avaliação da cárie dentária e Índice de MáOclusão, que classifica a oclusão em normal, presença de má-oclusão leve e moderada/ severa, respectivamente.

A análise de consistência do banco de dados e a análise descritiva dos dados foram realizadas utilizando o programa Epi-Info, versão 6.04. Para as análises estatísticas utilizou-se o programa MINITAB ${ }^{\circledR}$, especialmente a estatística " $t$ " para o cálculo de Intervalos de Confiança a 95\% do ceo-d das localidades avaliadas, e a estatística ANOVA para comparação de médias.

\section{Resultados}

Um total de 1374 crianças foi examinado em todos os DS, dos quais $682(49,60 \%)$ eram do sexo masculino e $692(50,4 \%)$ do sexo feminino. A Tabela 1 apresenta a média e os intervalos de confiança a 95\% do ceo-d por distrito sanitário, assim como dos seus componentes. O valor global de ceo-d da população estudada foi igual a 1,97 (IC de 95\% 1,81$2,12)$. Os mais altos valores do ceo-d foram observados nos DS de Brotas, Centro Histórico, Pau da Lima, Itapuã e Itapagipe, não se observando diferenças estatisticamente significantes $(p>0,05)$.

\section{Tabela 1}

Média de dentes cariados, de dentes extraídos, de dentes obturados e ceo-d com seus respectivos Intervalos de Confiança a 95\% de crianças com 05 anos, por Distrito Sanitário. Salvador, Bahia, 2005.

\begin{tabular}{lrcccc}
\hline \multirow{2}{*}{ Distrito } & $\mathbf{N}$ & cariados & extraídos & obturados & ceo-d (IC95\%) \\
\cline { 3 - 4 } & & Média & Média & Média & \\
\hline & & & & & \\
Centro Histórico & 40 & 2,33 & 0,10 & 0,15 & $2,58(1,42-3,73)$ \\
Itapagipe & 80 & 1,71 & 0,11 & 0,28 & $2,10(1,51-2,69)$ \\
São Caetano-Valéria & 80 & 1,40 & 0,21 & 0,04 & $1,60(1,13-2,07)$ \\
Liberdade & 40 & 1,03 & 0,03 & 0,33 & $1,38(0,59-2,16)$ \\
Brotas & 40 & 3,38 & 0,05 & 0,30 & $3,73(2,36-5,10)$ \\
Barra - Rio Vermelho & 115 & 1,40 & 0,02 & 0,31 & $1,73(1,21-2,25)$ \\
Boca do Rio & 78 & 1,10 & 0,04 & 0,05 & $1,19(0,74-1,64)$ \\
Itapuã & 70 & 2,06 & 0,27 & 0,09 & $2,41(1,62-3,20)$ \\
Pau da Lima & 40 & 2,28 & 0,13 & 0,08 & $2,46(1,64-3,31)$ \\
Cabula - Beiru & 40 & 1,60 & 0,05 & 0,25 & $1,90(1,04-2,76)$ \\
Subúrbio Ferroviário & 646 & 1,76 & 0,07 & 0,22 & $2,03(1,81-2,25)$ \\
Cajazeiras & 105 & 1,12 & 0,03 & 0,30 & $1,45(0,94-1,96)$ \\
Total & 1374 & 1,68 & 0,07 & 0,20 & $1,97(1,81-2,12)$ \\
\hline
\end{tabular}

Comparação das médias do ceo-d (ANOVA) p>0,05. 
Do total de dentes atacados pela cárie (2700), $2316(85,8 \%)$ apresentavam cavidade, $115(4,3 \%)$ haviam sido extraídos e 288 (10,7\%) estavam obturados (Tabela 2). A prevalência de cárie dentária foi de $49,6 \%$, enquanto que $50,4 \%$ apresentaram-se livres de cárie, isto é, com ceo-d=0.

Em relação às afecções gengivais, apenas 102 indivíduos (7,4\%) apresentaram alterações (Tabela 2). Quanto à freqüência de maloclusão, $720(52,4 \%)$ crianças apresentavam oclusão normal, 342(24,9\%) maloclusão leve e 175 maloclusão moderada ou grave (12,7\%), de acordo com os critérios do índice de maloclusão utilizados. ${ }^{8}$

Tabela 2

Prevalência de dentes cariados, extraídos e obturados, maloclusão e alteração gengival em indivíduos de cinco anos. Salvador, Bahia, 2005.

\begin{tabular}{lrc}
\hline & N & $\%$ \\
\hline ceo-d & & \\
Dentes cariados & 2316 & 85,8 \\
Dentes extraídos & 115 & 4,3 \\
Dentes obturados & 288 & 10,7 \\
Total de dentes atacados pela cárie examinados & 2719 & 100,0 \\
Maloclusão* & 720 & 52,4 \\
Sem alteração & 342 & 24,9 \\
Leve & 175 & 12,7 \\
Moderada/Grave & & \\
Alteração Gengival & 1272 & 7,4 \\
Sem alteração & 102 & \\
Com alteração & & \\
\hline
\end{tabular}

* Sem informação=137.

\section{Discussão}

A prevalência da cárie dentária entre as crianças de cinco anos estudadas foi de $49,6 \%$ e $50,4 \%$ apresentavam-se livres desta doença, denotando que a meta estabelecida pela OMS para o ano 2000 de $50 \%$ das crianças de cinco a seis anos livres de cárie, foi atingida no município. Essa prevalência de crianças livres de cárie mostrou-se mais elevada do que a observada na Região Nordeste no inquérito nacional realizado em 2003,3 e igualmente mais elevada do que a prevalência encontrada em crianças aos seis anos de idade dos municípios de Blumenau10 e Pelotas, ${ }^{4}$ ambos localizados na Região Sul do Brasil. Percentuais semelhantes de crianças pré-escolares livres de cárie foram encontrados por Leite e Ribeiro ${ }^{13}$ (50,6\%), no Estado de Minas Gerais, e por Moura et al. ${ }^{6}(56,9 \%)$ no Estado do Piauí.

O percentual de dentes cariados entre aqueles atacados pela cárie do índice ceo-d na população do presente estudo $(85,8 \%)$ foi igualmente inferior ao índice encontrado no Nordeste $(91,3 \%){ }^{3}$ No entanto, o percentual de dentes extraídos do índice ceo-d na população deste estudo (4,3\%) foi mais elevado do que observado na Nordeste $(3,7 \%) .3$ Observou-se um percentual de $10,7 \%$ de dentes obturados do índice ceo-d, resultado superior ao do Nordeste $(4,7 \%){ }^{3}$ Esses resultado evidenciam, portanto, a elevada necessidade de tratamento restaurador devido à cárie e igualmente a necessidade de tratamento mutilador em indivíduos de tão tenra idade nessa população. É historicamente reconhecido que as políticas públicas de saúde bucal no Brasil estiveram particularmente voltadas para a população de escolares, as quais têm resultado na melhoria das condições de saúde bucal dessa população quando comparada à população de pré-escolares, adultos e idosos. 18 Atualmente, com a expansão da estratégia do PSF, um modelo assistencial reorientador das práticas da atenção básica, é possível que, a partir do desenvolvimento de intervenções integradas da saúde da criança, as ações de saúde bucal tornem-se mais efetivas na melhoria das condições de saúde bucal dessas populações.

Os resultados deste estudo aproximam-se da realidade epidemiológica de saúde bucal das 
crianças de cinco anos residentes áreas de cobertura do PSF e assistidas pelo de Procedimentos Coletivos de Saúde Bucal de Salvador. Além de gerar informação para intervenção, o inquérito permitiu a incorporação da metodologia de levantamentos de saúde bucal, recomendada pela OMS, pelos serviços de saúde. Assim, os valores da média e composição percentual do ceo-d estratificados por DS são representativos da situação de cada área de abrangência pesquisada, podendo contribuir para o planejamento e programação das ações a serem desenvolvidas nas localidades estudadas.

A gravidade da cárie dentária, expressa pelos valores de ceo-d, não foi maior nas localidades onde as ESB atuam há mais tempo, como nos DS Subúrbio Ferroviário e Barra Rio Vermelho. Esses resultados provavelmente se devem às ações educativas, preventivas e curativas implementadas nestes locais.

A prevalência de alteração gengival $(7,4 \%)$ encontrada foi mais elevada do que a prevalência da região Nordeste $(6,5 \%)$, encontrada no SB Brasil. 3 Silveira et al. 19 avaliando o índice de sangramento gengival em crianças com média de idade de nove anos inseridas em um programa de promoção da saúde no Rio de Janeiro, encontrou um índice de $13 \%$ na primeira avaliação e de $5 \%$ ao final do estudo, revelando melhora do estado periodontal. Gomes Filho et al.,20 ao avaliarem a condição gengival de 300 crianças de quatro a seis anos de idade participantes de um programa de promoção de saúde bucal do município de Ferira de Santana, Bahia, concluíram que houve uma prevalência de $4,4 \%$ de sítios com sangramento gengival quando realizaram a sondagem periodontal, sendo que houve uma tendência ao aumento de sítios sangrantes com o aumento da idade. Cypriano et al. ${ }^{9}$ encontraram uma prevalência de $68,6 \%$ de sangramento gengival em crianças de cinco anos no município de Piracicaba, São Paulo, avaliados através do IPC quanto à presença de sangramento e cálculo dental. Os resultados do presente estudo corroboram achados do estudo de Silveira et al. 19 e Gomes Filho et al. ${ }^{20}$ No entanto, esta comparação deve ser analisada com cautela, pois os índices utilizados foram diferentes, assim como o tamanho da amostra e desenhos de estudos.

Quanto à prevalência de maloclusões, verificouse que $52,4 \%$ das crianças apresentaram oclusão normal, um percentual inferior ao observado no SB Brasil 3 da região Nordeste $(57,7 \%)$. A ocorrência de maloclusão leve foi maior em Salvador $(24,9 \%)$ quando comparada à região Nordeste $(22,7 \%)$, onde também foi observada maior prevalência de malo- clusão moderada/severa (17,40\%). Outros estudos também avaliaram a maloclusão em localidades do Brasil e no mundo, tendo encontrado, assim como neste inquérito, alta proporção de crianças acometidas por problemas oclusais, os quais estão relacionados com problemas na fala, mastigação, estética e convívio social dos indivíduos. ${ }^{21} \mathrm{Em}$ Bauru, São Paulo, a prevalência de oclusopatias foi de 54,1\% em indivíduos da mesma faixa etária, ${ }^{22} \mathrm{e}$ em São Paulo, capital, foi de 49\%. ${ }^{23}$ Peres, ${ }^{24}$ encontrou uma prevalência de $46,3 \%$ de mordida aberta anterior em crianças de cinco anos de idade do município de Pelotas, Rio Grande do Sul. Nesse mesmo estudo foi observado que $18,2 \%$ dessas crianças eram portadoras de mordida cruzada posterior. Na Finlândia, Karjalainen et al. ${ }^{25}$ verificaram uma prevalência de $18 \%$ de mordida aberta anterior, $13 \%$ de mordida cruzada posterior e $18 \%$ de overject $>3 \mathrm{~mm}$ em crianças de três anos de idade. Esta alteração bucal está fortemente relacionada com hábitos bucais adquiridos precocemente na infância, como os hábitos deletérios de sucção de chupeta e dedo.2527

São poucas as informações epidemiológicas sobre alterações periodontais e de maloclusões em crianças na faixa etária pré-escolar no Brasil, por isso houve dificuldade em se comparar os resultados do presente inquérito com outros estudos nacionais já realizados. Daí, a importância de divulgar os resultados desse estudo que se originam de inquérito realizado em usuários da rede pública de saúde, e que podem contribuir para o delineamento de políticas de saúde que reduzam as desigualdades em saúde no município.

Entretanto, deve-se ressaltar que tais resultados necessitam ser interpretados com cuidado, tendo em vista as limitações metodológicas advindas do processo de amostragem, que não permite estimativas para a população de Salvador nessa faixa etária. O fato do estudo não ter abrangido todas as áreas do município e de não se ter realizado a calibração intraexaminador pode ter resultado em vieses de seleção e aferição na análise. Entretanto, as comparações entre os estudos permitiram observar semelhanças e disparidades da situação de saúde bucal local em relação aos dados encontrados na Região Nordeste. Os resultados desse estudo igualmente revelam a elevada ocorrência de problemas de saúde bucal nas crianças estudadas, a maioria preveníveis e controláveis, tais como a cárie dentária, as alterações gengivais e as maloclusões, os quais podem interferir negativamente no desenvolvimento físico e emocional dos pré-escolares acometidos.28 Para controlá-los e minimizá-los são 
necessárias ações preventivas, educativas e curativas mais efetivas, a serem desenvolvidas pelos serviços de saúde, especialmente na atenção primária, que

\section{Referências}

1. Brasil. Ministério da Saúde. Levantamento epidemiológico de saúde bucal: Brasil, zona urbana, 1986. Brasília, DF; 1988 .

2. Oliveira AGRC. Levantamento epidemiológico em saúde bucal - cárie dental. Araçatuba; 1998. (monografia online). (acesso em: 21 set. 2005). Disponível em: http://planeta. terra.com.br/saude/ngelonline/artigos/art_epid/epi_96.pdf.

3. Brasil. Ministério da Saúde. Projeto SB Brasil 2003: condições de saúde bucal da população brasileira 20022003. Resultados principais. Brasília, DF; 2004.

4. Peres MA, Latorre MRD, Sheiham A, Peres KG, Barros FC, Hernandez PG, Mass AMN, Romano AR, Victora CG. Determinantes sociais e biológicos da cárie dentária em crianças de seis anos de idade: um estudo transversal aninhado numa coorte de nascidos vivos no sul do Brasil. Rev Bras Epidemiol. 2003; 6: 293-306.

5. Tomita NE, Bijella VT, Lopes ES, Franco LJ. Prevalência da cárie dentária em crianças da faixa etária de 0 a 6 anos matriculados em creches: importância de fatores socioeconômicos. Rev Saúde Pública. 1996; 30: 413-20.

6. Moura LFAD, Moura MS, Toledo OA. Dental caries in children that participated in a dental program providing mother and child care. J Appl Oral Sci. 2006; 14: 53-60.

7. Saito SK, Deccico HMU, Santos MN. The effect of infant feeding practices and associated factors on dental caries in preschool children, ages form 18 up to 48 months. Rev Odontol. Univ São Paulo. 1999; 13: 5-11.

8. Tomita NE, Nadanovsky P, Vieira ALF, Lopes ES. Taste preference for sweetness and caries prevalence preschoolchildren. Rev Saúde Pública. 1999; 33: 542-6.

9. Cypriano S, Sousa MLR, Rihs LB, Wada RS. Oral health among preschool children in Brazil. Rev Saúde Pública. 2003; 37: 247-53.

10. Traebert JL, Peres MA, Galesso ER, Zabot NE, Marcenes W. Prevalência e severidade da cárie dentária em escolares de seis e doze anos de idade. Rev Saúde Pública. 2001; 35 : 283-8.

11. Freire MCM, Pereira MF, Batista SMO, Borges MRS, Barbosa MI, Rosa AGF. Prevalência de cárie e necessidade de tratamento em escolares de seis a doze anos de idade, Goiânia, GO, Brasil, 1994. Rev Saúde Pública. 1997; 31 : 44-52.

12. Manfredini MA. Planejamento em saúde bucal. In: Pereira AC. Odontologia em Saúde Coletiva. Planejando ações e promovendo saúde. São Paulo: Artmed; 2003. p. 50-63.

13. Leite ICG, Ribeiro RA. Dental caries in the primary dentition in public nursery school children in Juiz de Fora, Minas Gerais, Brazil. Cad Saúde Pública. 2000; 16: 71722.

Recebido em 15 de outubro de 2008

Versão final apresentada em 8 de julho de 2009

Aprovado em 23 de julho de 2009 possam contribuir a melhoria da condição de saúde bucal nesse grupo.

14. Barros SG, Castro Alves A, Pugliese LS, Reis SRA. Contribuição ao estudo da cárie dentária em crianças de 030 meses. Pesq Odontol Bras. 2001; 15: 215-22.

15. Fédèration Dentaire Internationale. Global goals for oral health in the year 2000. Int Dent J.1982; 32: 74-7.

16. IBGE (Instituto Brasileiro de Geografia e Estatística). População de Salvador. (online) [acesso em: 2 jul 2009) Disponível em: http://www.ibge.gov.br.

17. OMS (Organização Mundial da Saúde). Levantamento epidemiológico básico de saúde bucal: manual de instruções. 4. ed. São Paulo: Santos; 1999.

18. Pereira ACP. Odontologia em saúde coletiva. São Paulo: Artmed; 2003.

19. Silveira JLGC, Oliveira V, Padilha WWN. Evaluation of the reduction of the visible plaque index and of gum bleeding index in a program of oral health promotion for children. Pesq Odontol Bras. 2002; 16: 169-74.

20. Gomes Filho IS, Miranda DAO, Trindade SC, Santos CTS, Freitas COF, Cruz SS, Macedo TCN, Passos JS. Relationship among gender, race, age, gingival width and probing depth in primary teeth. J Periodontol. 2006; 77: 1032-42.

21. Spilä K, Zitting P, Laukkanen P, Järvelin MR, Oikarinen KS, Raustia, AM. Temporomandibular disorders, occlusion, and neck pain in subjects with facial pain: a case-control study. J Craniomand Pract. 2002; 20: 158-64.

22. Tomita NE, Bijellla V, Franco LJ. The relationship between oral habits and malocclusion in preschool children. Rev Saúde Pública. 2000; 34: 299-303.

23. Frazão P, Narvai PC, Latorre MRDO, Castellanos RA. Malocclusion prevalence in the deciduous and permanent dentition of schollchildren in the city of São Paulo, Brazil, 1996. Cad Saúde Pública. 2002; 18: 1197-205.

24. Peres MA. Oclusopatias na dentição decídua: acúmulo de riscos do nascimento à primeira infância [tese]. São Paulo: Faculdade de Saúde Pública da Universidade de São Paulo; 2002.

25. Karjalainen S, Ronning O, Lapinleimu H, Simell H. Association between early weaning, non-nutritive sucking habits and occlusal anomalies in 3 year-old Finnish children. Int J Paediatr Dent. 1999; 9: 169-73.

26. Svedstrom-Oristo, AL, Pietilä T, Pietilä I, Aalanen P, Varrela J. Outlining the morphological characteristics of acceptable occlusion. Community Dent Oral Epidemiol. 2000; 28: 35-41.

27. Kharbanda PP, Sidhu SS, Shukla DK, Sundaran KR. A study of the etiological factors associated with de development of malocclusion. J Clin Ped Dent. 1994; 18: 95-8.

28. Reisine S, Douglas JM. Psychosocial and behavioral issues in early chilhood caries. Community Dent Oral Epidemiol. 1998; 26 (Suppl. 1): 32-44. 